\title{
Anger among Adolescents: The Role of Mindfulness
}

\author{
Ashu Dhawan ${ }^{1}$, Sandeep Singh $^{2}$ and Taruna ${ }^{3 *}$ \\ ${ }^{1}$ Research Scholar, ${ }^{2}$ Professor \& Head, ${ }^{3}$ Assistant Professor, Department of Applied Psychology, Guru Jambheshwar University of Science Technology, \\ Hisar, Haryana, India
}

Received 06 Nov 2017, Accepted 10 Jan 2018, Available online 18 Jan 2018, Vol.6 (Jan/Feb 2018 issue)

\begin{abstract}
Adolescence is considered as the transitional stage of development wherein the transformation from childhood to adulthood takes place. This is the period of optimum health accompanied with biological and psychological changes. Along with various emotional changes among adolescents anger has been reported to have serious consequences than any other emotion. Anger is mainly responsible for hostility and violence (Pullen, 2015). The research studies have constantly explored the causative factors of anger in this developmental age group. The present research study made an attempt to explore the relationship between the two dimensions of anger namely, the state anger and the trait anger along with the trait of mindfulness. The sample of the present study was comprised of 200 adolescents $(N=200)$. StateTrait Anger Expression Inventory (STAXI-2, Spielberger, 1996) and Mindful Attention Awareness scale (MAAS, trait version, Brown and Ryan, 2007) were used to measure the variables of the study. Findings of the present study revealed the significant negative correlation ( $r=-.176<.01)$ between the trait of mindfulness and trait anger. Furthermore, the findings also established the significant positive correlation between state anger and trait anger $(r=.518<.05)$. No gender difference for the trait of mindfulness and anger was reported in the study.
\end{abstract}

Keywords: State Anger, Trait Anger, Mindfulness

\section{Introduction}

Adolescence is known as the transitional stage of development between childhood and adulthood. There are different opinions about period of adolescence; these may vary from culture to culture ranging from the age between 12 years to 19 years. Adolescence is the period of maximal biological as well as psychological changes. When it comes to the psychological changes, the emotional changes are observable and reported to be at their peak. Anger is such an emotion the expression of which may land adolescents into trouble and even more serious situations. Anger is the subjective experience of an emotion (Feindler, 1994). It has further been defined as "an emotional state that consists of feelings that vary in intensity, from mild irritation or annoyance to intense fury and rage."

Anger in youth is one of the major causes for various mental health problems worldwide. If not controlled, it is a major contributory factor for deaths due to homicide, suicide and other injuries (CDC, 2015). Anger is also pushed up by personality characteristics of individuals. Adolescents with high dispositional anger have exhibited negative health, social and academic consequences related to anger (Colleen, 2015). Study further revealed

*Corresponding author's ORCID ID: 0000-0003-1976-2817 DOI: https://doi.org/10.14741/ijmcr.v6i01.10911 that trait anger leads to higher risk-taking behavior in adolescents.

There is no dearth of researches indicative of the causative factors behind the anger among adolescents. Researchers have constantly tried to explore the personality traits that could be attributed to the anger among adolescents. Among various causative factors researchers have tried to study the anger as a trait and state as well. Deliberating upon the various causative factors of anger researchers are trying to explore whether the trait of mindfulness plays its role in anger or not.

Kabat-Zinn (1994) described mindfulness as "paying attention in a particular way: On purpose, in the present moment, and that too nonjudgmentally." Researchers have also described mindfulness as a metacognitive state of detached awareness (Teasdale,1999). Metacognitive processing which is not only limited upto acquisition of knowledge regarding one's cognition, but is also extended to regulatory processes such as planning, monitoring, and evaluating. Mindfulness is concerned with whole existence and personality of individual simultaneously and considers him/her as an integrated whole. Mindfulness works in parallel like interactive cognitive subsystems that works on physical and sensational effects and individual's thoughts, emotions and excitements simultaneously.

$80 \mid$ Int. J. of Multidisciplinary and Current research, Vol.6 (Jan/Feb 2018) 
The incident rate of anger among adolescents is increasing day by day, leading to behavioral issues of serious nature. The present study conceptualises to explore the relationship between trait of mindfulness and anger. The study also aims to explore gender differences in the trait of mindfulness and anger.

\section{Objectives}

1. To study the relationship between mindfulness and anger among adolescents.

2. To study the gender differences in mindfulness and anger among adolescents.

\section{Hypotheses}

1. There shall be significant inverse relationship between mindfulness and anger among adolescents.

2. There shall be significant gender differences in mindfulness and anger among adolescents.

\section{Method}

\section{Sample}

The target population of the present research study was adolescents with the age group of $16-19$ years. The sample for the present research comprises of 200 $(N=200)$ adolescents. The sample is further categorized on the basis of gender (Male=100 \& Female=100) from the state of Haryana, India.

\section{Measures}

The measures used in the study were selected in accordance with the objective of the study.

1. State-Trait Anger Expression Inventory (STAXI2; Spielberger, 1996): The STAXI-2 is a 49-item questionnaire containing two subscales namely, State Anger Scale and Trait Anger scale. The dimensions of subscales assess state anger and trait anger respectively. The test possesses good psychometric properties with the reliability at the Chronbach's alpha values ranging from .71 to .89 for the trait and state scales respectively. 2 . Mindful Attention Awareness scale (MAAS, trait version; Brown \& Ryan, 2007): The MAAS is a 15 item scale designed to assess the trait of mindfulness as a receptive state of mind in which attention, informed by a sensitive awareness of what is occurring in the present, simply observes what is taking place. It is a short item scale which takes five minutes or less to complete. The scale possesses good psychometric properties with internal consistency levels (Chronbach's alphas) ranging from .80 to .90. Along with this the scale has also demonstrated the high discriminant and convergent validity, knowngroup validity, and criterion validity.

Procedure
After establishing the rapport with the subjects, the participants were briefed about the nature and purpose of study. The dimensions of state and trait anger were studied with the help of STAXI-2 and the trait of mindfulness was assessed with the help of MAAS. The scales were administered individually on each and every participant. The scores on the respected measures were obtained as per the scoring standards and the data obtained was put to statistical analysis.

\section{Statistical Analysis}

The data obtained was put to statistical analysis and inferences were drawn. Descriptive statistics (mean and standard deviation) and Pearson's product moment coefficient of correlation were applied to explore the correlation among the variables. Independent t- test was further applied to analyze the mean differences between the gender (Male and Female).

\section{Results}

The data obtained from the present study was analyzed with the help of SPSS 16.0. The descriptive statistics (mean and standard deviation) and Pearson's product moment coefficient of correlation was applied to explore the relationship among the variables of the present study. The independent $t$-test was further applied to study the mean difference between the genders.

Table 1 Descriptive statistics and intercorrelation matrix $(N=200)$

\begin{tabular}{cccccc}
\hline Variables & Mean & $\begin{array}{c}\text { Standard } \\
\text { Deviation }\end{array}$ & Mindfulness & $\begin{array}{c}\text { State } \\
\text { Anger }\end{array}$ & $\begin{array}{c}\text { Trait } \\
\text { Anger }\end{array}$ \\
\hline Mindfulness & 52.57 & 14.10 & 1 & -.062 & $-.176^{*}$ \\
State Anger & 24.85 & 6.30 & 1 & $.518^{* *}$ \\
Trait Anger & 20.66 & 5.34 & & 1 \\
\hline \multicolumn{5}{c}{${ }^{* *}$ Correlation is significant at 0.01 level } \\
& $*$ Correlation is significant at 0.05 level
\end{tabular}

Table 2 Descriptive statistics for the gender differences on the state anger, trait anger and mindfulness

\begin{tabular}{cccccc}
\hline \multirow{2}{*}{ Variable } & \multirow{2}{*}{$\mathrm{N}$} & \multicolumn{2}{c}{ Male } & \multicolumn{2}{c}{ Female } \\
& & Mean & SD & Mean & SD \\
\hline Mindfulness & 100 & 52.20 & 14.84 & 52.95 & 13.39 \\
State Anger & 100 & 25.30 & 6.97 & 24.41 & 5.56 \\
Trait Anger & 100 & 20.47 & 5.87 & 20.86 & 4.78 \\
\hline
\end{tabular}

Table 3 Outcomes for the gender difference on the state anger, trait anger and mindfulness (Independent samples t-test)

\begin{tabular}{|c|c|c|c|c|c|c|}
\hline Variable & Gender & $\mathrm{N}$ & $\begin{array}{c}\text { Mean } \\
\text { Differences }\end{array}$ & $\mathrm{t}$ & $d f$ & $\begin{array}{l}\text { p(Sig.2- } \\
\text { tailed) }\end{array}$ \\
\hline \multirow{2}{*}{ Mindfulness } & Male & 100 & \multirow{2}{*}{-.750} & 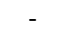 & \multirow{2}{*}{198} & \multirow{2}{*}{.708} \\
\hline & Female & 100 & & .375 & & \\
\hline \multirow{2}{*}{ State Anger } & Male & 100 & \multirow{2}{*}{.890} & \multirow{2}{*}{.998} & \multirow{2}{*}{198} & \multirow{2}{*}{.320} \\
\hline & Female & 100 & & & & \\
\hline \multirow{2}{*}{ Trait Anger } & Male & 100 & \multirow{2}{*}{-.390} & \multirow{2}{*}{.515} & \multirow{2}{*}{198} & \multirow{2}{*}{.607} \\
\hline & Female & 100 & & & & \\
\hline
\end{tabular}

81 | Int. J. of Multidisciplinary and Current research, Vol.6 (Jan/Feb 2018) 
Findings obtained from the present study (Table 1) depict the significant positive correlation between state anger and trait anger $(r=.518<.01)$. The findings have also established negative correlation between $(r=-.062)$ between the trait of mindfulness and state anger.

The findings obtained from the present research study further depict the significant negative correlation between $(r=-.176<.05)$ between mindfulness and trait anger.

The results (Table 3 ) further revealed that there is no gender difference in the scores for the dimension of state anger, trait anger and mindfulness.

\section{Discussion}

The present research study aimed to explore the relationship between the trait of mindfulness and anger (state anger and trait anger).Consistent with our first hypothesis the findings of the present study depict the significant negative correlation between trait anger and the trait of mindfulness. Therefore, it can be inferred that adolescents who are high on the trait of mindfulness are low on the trait anger. The obtained findings are in line with the research study conducted by Heppner et al., (2008) wherein they found mindfulness to be negatively associated with anger and hostile attribution. They further concluded that mindfulness reduces anger among psychotherapist in training and individuals in correctional settings.

Research in the past has also found that mindfulness enhances the individual's ability of paying attention in the present moment which enables them to regulate their attention in the present moment resulting in decreased rumination and worry, allowing experiences to naturally come and go without trying to remove any unpleasant emotion or engaging in reactive behavior (Segal, et al., 2002).

The present research study provides the understanding of the fact that the trait of mindfulness enables adolescents to be more aware about their feelings consequent to which they become habitual to see their thoughts and emotions, the reactivity gets reduced automatically. As mindfulness results in feeling detached from the various events and hence just focussing on "here and now" approach (Wilde, 2001). It was also found that high dispositional mindfulness was associated with lower levels of anger, among criminal justice professionals (Kelley \& Lambart, 2012).

The findings obtained from the present study established the positive correlation between state anger and trait anger even though the correlation is statistically not significant but provides the understanding that if an individual is high at dispositional anger will also exhibit high reactivity and anger while in sensitive states.

Mindfulness has also been associated with positive feelings like well-being, joy and compassion. All of these attributes are thought to decrease the reactivity level and help in reducing anger (Arch and Craske, 2006).
The present study didn't find any significant gender difference among adolescents on the trait of mindfulness and anger.

\section{Conclusion}

Anger can be problematic in a diverse array of situations like mental health, physical health, school, home, and workplace, etc, consequences of which have been reported to be harmful for peers, family, colleagues and society as well. The present study documents the significant inverse correlation between mindfulness and anger. There are various treatments that have documented their efficacy in reducing anger among adolescents e.g., functionally derived behavioral contingencies and cognitive behavior therapy (Brosnan \& Healy, 2011).

Present research study marks the attention of the health and mental health professional working in the area that by working on developing the skill of mindfulness the trait anger can be reduced among adolescents. Mindfulness is aimed at reducing anger by providing cognitive skills for managing anger without reliance on other individual. Based on the findings of the present study it is suggested that randomized control studies with mindfulness based therapies may be conducted to compare with other treatments and therapies aimed at reducing anger among adolescents and other age groups. Hence, there exists the need to make intervention based studies in future. Further studies need to be carried out with bigger sample in order to determine specific causal mechanisms behind anger among adolescents.

\section{References}

[1] Allen, M., Bromley, A., Kuyken, W., \& Sonnenberg, S. J. (2009). Participants' experiences of mindfulness-based cognitive therapy: "it changed me in just about every way possible". Behavioral and Cognitive Psychotherapy, 37, 413e430.

[2] Arch JJ, Craske MG. Mechanisms of mindfulness: emotion regulation following a focused breathing induction. Behavior Research and Therapy.2006;44(12):1849-1858.

[3] Brosnan, J. \& Healy ,O.(2011) A review of behavioral interventions for the treatment of aggression in individuals with developmental disabilities. Research in Developmental Disabilities 32,437-446.

[4] Brown, K. W., Ryan, R. M., \& Creswell, J. D. (2007) Mindfulness: Theoretical Foundations and Evidence for its Salutary Effects. Psychological Inquiry, 18, 211 e-237.

[5] CDC. 2006. Centers for Disease Control [CDC]. Youth risk behavior surveillance - United States, 2005 Morbidity and Mortality Weekly report, 55 (SS-5). 2006. Retrieved March 23, 2007. http://www.cdc.gov/mmwr/PDF/SS/SS5505.pdf

[6] Colleen, A.Q. 2015. Colleen A. Quinn, David Rollock, and Scott R. Vrana 'A Test of Spielberger's State-Trait Theory of Anger with Adolescents: Five Hypotheses' NIH Public Access, Emotion. 2014 February, 14(1): 74-84. doi:10.1037/a0034031.

[7] Feindler, E. L., \& Ecton, R. B. (1994). Adolescent anger control: Cognitive behavioral techniques. Needham Heights, MA: Allyn \& Bacon.

82 | Int. J. of Multidisciplinary and Current research, Vol.6 (Jan/Feb 2018) 
[8] Feldman, G. C., Hayes, A. M., Kumar, S. M., Kamholz, B. W., Greeson, J. M., \& Laurenceau, J. (2005, November). Assessing mindfulness in the context of emotion regulation: The Revised Cognitive and Affective Mindfulness Scale (CAMS-R). Paper presented at the 39th Annual Convention of the Association for Behavioral and Cognitive Therapies, Washington, DC.

[9] Hayes, S. C., Follette, V. M., \& Linehan, M. M. (Eds.). (2004). Mindfulness and acceptance: Expanding the cognitivebehavioral tradition. New York: Guilford Press.

[10] Heppner,W. L., Kernis, M. H., Lakey, C. E., Campbell,W. K., Goldman, B. M., Davis, P. J., \& Cascio, E. V. (2008). Mindfulness as a means of reducing aggressive behavior: dispositional and situational evidence. Aggressive Behavior, 34(5), 486-496.

[11] Kabat-Zinn J. Wherever You Go, There You Are: Mindfulness Meditation in Every Day Life. New York: Hyperion; 1994.

[12] Kelley, T.M.,\& Lambert, E. G. (2012).Mindfulness as a potential means of attenuating anger and aggression for prospective criminal justice professionals. Mindfulness, 3(4), 261-274.

[13] Ortner, C. N., \& Zelazo, P. D. (2014). Responsiveness to a mindfulness manipulation predicts affect regarding an angerprovoking situation. Canadian Journal of Behavioral Science/Revue canadienne des sciences du comportement, 46(2), 117.
[14] Peters, J. R., Eisenlohr-Moul, T. A., Upton, B. T., \& Baer, R. A. (2013). Nonjudgment as a moderator of the relationship between present centered awareness and borderline features: synergistic interactions in mindfulness assessment. Personality and Individual Differences,55(1), 24-28

[15] Pullen, L. 2015. Pullen L, Modrcin MA, McGuire SL, Lane K, Kearnely M, Engle S.; Anger in Adolescent Communities: How Angry Are They?; Pediatr Nurs., 41(3): 135-40.

[16] Schraw G. Promoting general metacognitive awareness. Instr Sci. 1998;26:113-25.

[17] Segal Z, Williams JM, Teasdale J: Mindfulness-Based Cognitive Therapy for Depression. NewYork, Guilford, 2002.

[18] Sharma, M.K. 2012. Sharma MK, LNSuman, Pratima Murthy and Palaniappan Marimuthu, State-Trait Anger and Quality of Life Among Alcohol Users, German J. Psychiatry.

[19] Spielberger, C. D. (1996). State-trait anger expression inventory: Professional manual. Odessa, FL: Psychological Assessment Resources.

[20]Teasdale JD. Metacognition, mindfulness and the modification of mood disorders. Clin Psychol Psychother. 1999;6:146-55.

[21] Wilde, J. (2001). Interventions for children with anger problems. Journal of Rational-Emotive \& Cognitive-Behavior Therapy, 19 (3),191-197. 